\title{
Effectiveness of Reporting System for Carbapenem Antibiotics in Restricting Their Use
}

\author{
Yuichi Koizumi ${ }^{11}$, Tomo Nakanishi ${ }^{1}$, Mami Nomura ${ }^{1}$, \\ Hajime Tanaka ${ }^{2}$, Atsuko Mugitani ${ }^{3}$, Shigekatsu Hatanaka ${ }^{4}$, \\ Yoichi Takahashi ${ }^{5}$ and Kenji Matsuyama ${ }^{6}$ \\ Department of Pharmacy ${ }^{1}$, Director of Fuchu Hospital ${ }^{2}$, Department of Hematology ${ }^{3}$, \\ Department of Clinical Laboratory ${ }^{4}$, Department of Infection Control ${ }^{5}$, Fuchu Hospital \\ School of Pharmaceutical Sciences, Mukogawa Women's University ${ }^{6}$ \\ $\left[\begin{array}{l}\text { Received February 7, } 2007 \\ \text { Accepted July 30, } 2007\end{array}\right]$
}

We designed a reporting system for the use of carbapenem antibiotics at Fuchu Hospital in order to restrict their use. We also examined the effect of the system on the number of vials of antibiotics used from December 2002 to November 2004 and compared the total amount of antibiotics used before and after introducing the system.

In our hospital, a large amount of panipenem is used, while the use of imipenem is limited. The antimicrobial use density (AUD) of panipenem decreased significantly from 9.85 to 7.59 due to the introduction of the reporting system $(p<0.01)$ while that of imipenem decreased from 0.06 to 0.04 . Therefore, the introduction of the system significantly influenced the use of panipenem but not that of imipenem. Following its introduction, the rate of occurrence of panipenem-resistant Pseudomonas aeruginosa decreased from $41.38 \%$ to $24.00 \%(p<0.001)$ while that of imipenem-resistant $P$. aeruginosa decreased from $18.23 \%$ to $10.91 \%(p<0.01)$. We therefore conclude that the reporting system brought about an apparent re duction in the occurrence of carbapenem-resistant $P$. aeruginosa.

Key words - carbapenem antibiotics, Pseudomonas aeruginosa, restricted use, antimicrobial use density, defined daily doses

\section{Introduction}

A series of broad-spectrum antibiotics have been introduced in clinical practice. However, the extensive use of antibiotics increases the risk of colonization and emergence of antibiotic-resistant strains such as methicillin-resistant Staphylococcus aureus (MRSA) that may cause clinical complications ${ }^{1,2)}$. Resistance to antipseudomonal antibiotics has increasingly become a problem in recent years following the emergence of a relatively high frequency of antimicrobial resistance during therapy ${ }^{3,4}$. Further, the extensive use of carbapenem antibiotics was reported to be one of the risk factors for the occurrence of multiple drug-resistant Pseudomonas aeruginosa (MDRP) infections ${ }^{5}$. In order to prevent the outbreak of such infections, an infection control team (ICT) comprising pharmacists, physicians, nurses, and medical technologists was formed in Fuchu Hospital in 1996. This team has surveyed the clinical use of all antibiotics, particularly the use of carbapenem antibiotics, by using a report system. Recently, the World Health Organization (WHO) has recommended the number of defined daily doses
(DDD) as a unit of measure for comparing antimicrobial use $^{6}$. A ntimicrobial use density (AUD) can be calculated us ing the DDD number ; this calculation is described in Methods. The AUD adjusts for the influence of the number of patients and number of days in the hospital. We calculated the AUD for the main antibiotics used by using the appropriate DDD number in the equation mentioned below (Methods, Calculation of the antimicrobial use density). The effect of restricting carbapenem use by the introduction of the re port system was verified by using the calculated AUD. We evaluated whether the introduction of the report system was able to reduce the occurrence of carbapenem-resistant $P$. aeruginosa. In this study, the pharmacy worked in conjunction with the ICT. The pharmacy determined the appropriate use of the antibiotics with the ICT.

The pharmacy proposed the introduction of the report sys tem at the infection control committee, and the report form was developed. The pharmacists proposed the method of operation of the report system. The operation method involved submitting the form to the pharmacy, ensuring the submis sion of the form to the pharmacy, and conveying the contents of the form to the ICT. If the doctor did not submit the 
form, the pharmacist did not give him the antibiotics. The pharmacist then called the doctor and explained the neces sity of submitting the form to him. Not submitting the form constituted a violation of the report system; preventing this violation is key to the success of this system. We attempted to eliminate this violation.

The purpose of this study is to decrease the inappropriate use of carbapenem antibiotics by the introduction of the re port system. In addition, we speculate that the decreased resistance of $P$. aeruginosa to carbapenem is related to the decreased use of this antibiotic.

\section{Methods}

\section{Experimental Procedure}

Fuchu Hospital provides medical treatment for acutephase patients; it has 380 beds. The hospital has a variety of treatment departments (cardiology, pulmonology, hepatology, hematology, general medicine, surgery, neurosurgery, cardiac surgery, orthopedic surgery, urology, obstetrics and gynecology, radiology, ophthalmology, otolaryngology, and dermatology). This study aimed to examine the antibiotics used to treat patients in Fuchu Hospital. The antibiotics examined during this study are listed in Table 1. All the antibiotics in the list are medicines used in our hospital.

When using carbapenem antibiotics, such as panipenem and imipenem, the physician in charge is required to fill the form depicted in Fig. 1. Unless this form is submitted to the Department of Pharmacy, the physician in charge cannot use carbapenem antibiotics. By analyzing these forms, the ICT can examine the usage of all carbapenem antibiotics. This report system has been introduced since December 2003. In

Table 1. List of the Antibiotics Examined in this Study.

\begin{tabular}{|l|lr|}
\hline Penicillin & Ampicillin (ABPC) & $1 \mathrm{~g}$ \\
& Ampicillin/Cloxacillin & $0.1 \mathrm{~g}$ \\
& Piperacillin (PIPC) & $1 \mathrm{~g}$ \\
& Ampicillin/Sulbactam (ABPC/SBT) & $1.5 \mathrm{~g}$ \\
\hline First-generation cephem & Cefazolin (CEZ) & $1 \mathrm{~g}$ \\
\hline Second-generation cephems & Cefmetazole (CMZ) & $1 \mathrm{~g}$ \\
& Cefotiam (CTM) & $1 \mathrm{~g}$ \\
& Flomoxef (FMOX) & $1 \mathrm{~g}$ \\
\hline Third-generation cephems & Cefoperazone (CPZ) & $1 \mathrm{~g}$ \\
& Ceftazidime (CAZ) & $1 \mathrm{~g}$ \\
\hline Fourth-generation cephems & Cefozopran (CZOP) & $1 \mathrm{~g}$ \\
& Cefpirome (CPR) & $1 \mathrm{~g}$ \\
\hline Monobactam & Aztreonam (AZT) & $1 \mathrm{~g}$ \\
\hline Amino glycoside & Isepamicin (ISP) & $0.2 \mathrm{~g}$ \\
& Gentamicin (GM) & $0.04 \mathrm{~g}$ \\
& Dibekacin (DKB) & $0.1 \mathrm{~g}$ \\
& Amikacin (AMK) & $0.2 \mathrm{~g}$ \\
& Streptomycin (SM) & $1 \mathrm{~g}$ \\
\hline Carbapenem & Panipenem/Betamipron (PAPM/BP) & $0.5 \mathrm{~g}$ \\
& Imipenem/Cilastatin (IPM/CS) * & $0.5 \mathrm{~g}$ \\
\hline Fosfomycin & Fosfomycin (FOM) & $1 \mathrm{~g}$ \\
\hline Tetracycline & Minocycline (MINO) & $0.1 \mathrm{~g}$ \\
\hline Lincomycin & Clindamycin (CLDM) & $0.6 \mathrm{~g}$ \\
\hline Macrolide & Erythromycin (EM) & $0.5 \mathrm{~g}$ \\
\hline New quinolone & Ciprofloxacin (CPFX) & $0.3 \mathrm{~g}$ \\
\hline Medicine for anti-MRSA & Arbekacin (ABK) & $0.1 \mathrm{~g}$ \\
& Vancomycin (VCM) & $0.5 \mathrm{~g}$ \\
\hline * Temporary usage & &
\end{tabular}

this study, the total number of vials of carbapenem antibiotics used, such as panipenem and imipenem, from December 2002 to November 2003 and from December 2003 to November 2004 was calculated, and the effect of intervention in their use was evaluated as AUD. In addition, the use of other antibiotics was examined in an identical manner.

\section{Calculation of the antimicrobial use density (AUD)}

The AUD for each main antibiotic was calculated on the basis of its DDD number that is defined by the WHO by us ing the following equation ${ }^{7,8)}$.

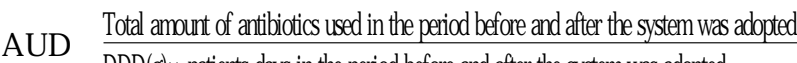
$\operatorname{DDD}(\mathrm{g}) \times$ patients days in the period before and after the system was adopted

$\times 1000$ (patient days)

The AUD for the main antibiotics that was calculated on the basis of the corresponding DDD number are summarized in Table 2.

\section{Evaluating susceptibility to the antibiotics}

The susceptibility to the antibiotics was classified as sensitive $(S)$, resistant $(R)$, and intermediate (I) based on the guidelines proposed by the National Committee for Clinical Laboratory Standards. Each examination was performed by

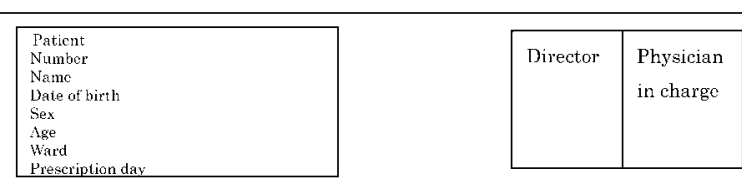

Report on the use of carbapenems

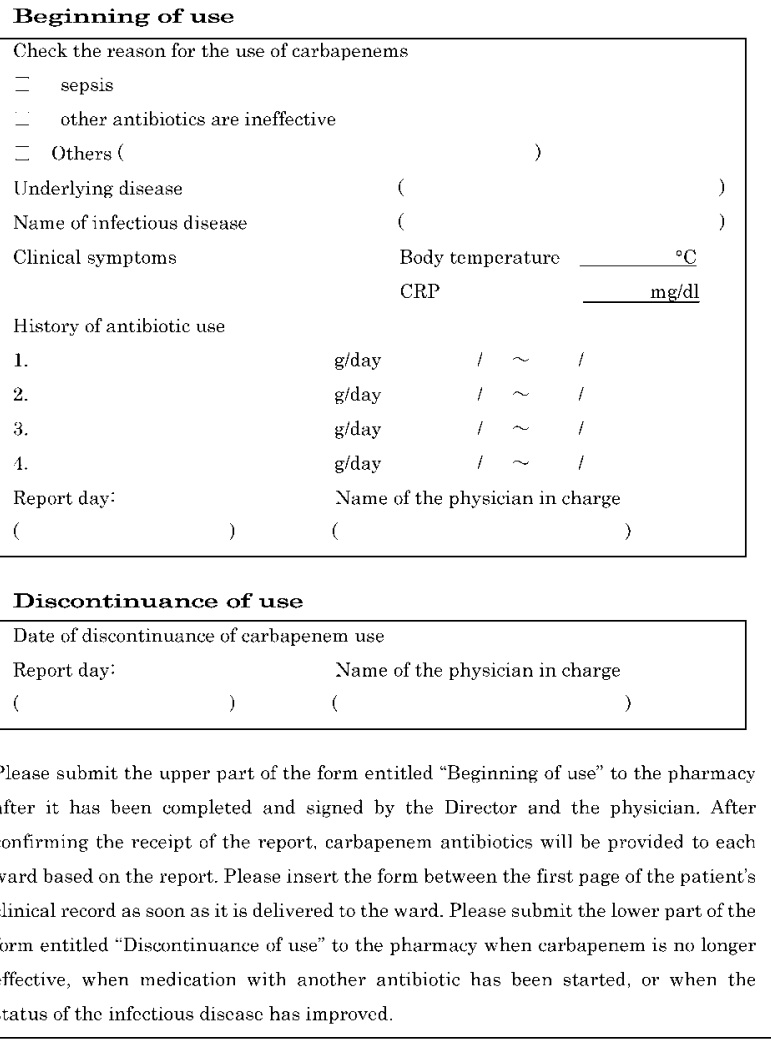

Fig 1 . Form Used to Report the Use of Carbapenems. 
using the disk method, and the automatic detector from Becton Dickinson was operated by the medical technologist at our hospital. The rates for the occurrence of resistant $P$. aeruginosa before and after the introduction of the report system were compared by using the susceptibility test for carbapenem antibiotics.

\section{Results and Discussion}

Fig. 2 shows the effect of the carbapenem report system on the total amount of all antibiotics (open circle) and the total amount of carbapenem antibiotics (closed square) used in terms of the total number of vials used during each half year from December 2002 to June 2003, July 2003 to No- vember 2003, December 2003 to June 2004, and July 2004 to November 2004. With regard to the total amount of antibiotics used, no significant effect was observed following the introduction of the report system. However, as shown in Fig. 2, the system caused a decrease in the use of carbapenem antibiotics. The AUD for panipenem was calculated by using the total amount of panipenem used each year be fore and after the introduction of the report system in the abovementioned equation (Methods, Calculation of the antimicrobial use density). The introduction of the report sys tem significantly reduced the AUD for panipenem from 9.85 to 7.59 ( $p<0.01$, Chi-square test) while that for imipenem decreased from 0.06 to 0.04 , i.e., no significant difference in its use was observed following the introduction of the report

Table 2. Comparison of the Overall A mount of A ntibiotics and the Antimicrobial Use Density (AUD) Used at Fuchu Hospital before and after the Implementa tion of the System.

\begin{tabular}{|l|r|r|r|r|c|}
\hline & $\begin{array}{c}\text { Before the introduction } \\
\text { of the system }\end{array}$ & $\begin{array}{c}\text { After the introduction } \\
\text { of the system }\end{array}$ & $\begin{array}{c}\text { Increase and } \\
\text { decrease }\end{array}$ \\
\cline { 2 - 6 } & toal amount & AUD & toal amount & AUD & \\
\hline Penicillin & 7169 & 7.35 & 7880 & 7.71 & $\uparrow$ \\
\hline The first generation cephem & 13728 & 34.61 & 12415 & 31.31 & $\downarrow$ \\
\hline The second generation cephem & 17239 & 32.6 & 17334 & 32.79 & $\uparrow$ \\
\hline The third generation cephem & 6414 & 12.13 & 7412 & 14.02 & $\uparrow$ \\
\hline The fourth generation cephem & 4111 & 7.77 & 5701 & 10.79 & $\uparrow$ \\
\hline Monobactam & 429 & 0.81 & 411 & 0.78 & - \\
\hline Amino glycoside & 1906 & 4.24 & 1439.5 & 3.20 & - \\
\hline Carbapenem & 5234 & 9.91 & 4044 & 7.63 & $\downarrow$ \\
\hline Fosfomycin & 3525 & 3.33 & 2083 & 1.97 & $\checkmark$ \\
\hline Tetracycline & 829 & 3.14 & 1353 & 5.12 & - \\
\hline Lincomycin & 3265 & 8.23 & 2444 & 6.16 & $\checkmark$ \\
\hline Macrolide & 217 & 0.82 & 216 & 0.82 & - \\
\hline New quinolone & 203 & 0.92 & 534 & 2.42 & - \\
\hline Medicine for anti-MRSA & 3024 & 5.72 & 2888 & 5.51 & $\downarrow$ \\
\hline
\end{tabular}

With regard to antibiotics of which more than 2000 vials used during the evaluation periods, we described the increase and decrease.

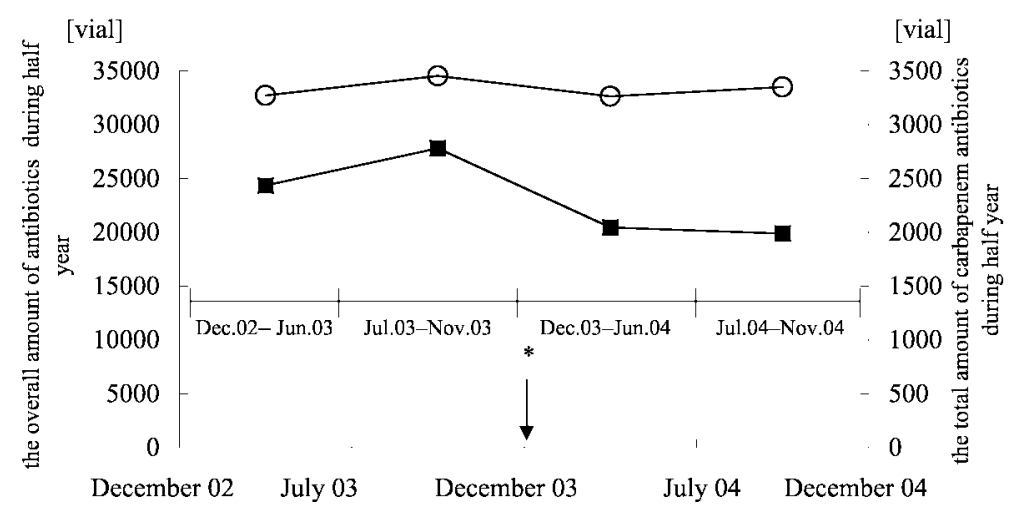

Fig 2. Changes in the Overall A mounts of A ntibiotics U sed and the total A mounts of Carbapenem Antibiotics used from December 2002 to November 2004.

The open circle represents the overall amount of antibiotics. The closed square represents the total amount of carbapenem antibiotics. An arrow represents the month when for the report system was introduced. 
system (Fig. 3).

Generally, carbapenem antibiotics are used to treat sepsis, severe infections, and refractory infections, and their use is recommended when other antibiotics are ineffective. However, physicians tend to prescribe carbapenem antibiotics even to those patients who do not fulfill the abovementioned criteria; this results in the emergence of carbapenemresistant $P$. aeruginosa and also MDRP. Our report system compels physicians to specify the reason for the choice of carbapenem in the form and therefore restricts the excessive use of carbapenem antibiotics. (Fig. 3)

The total number of antibiotic vials used from December 2002 to November 2004 and the A UD before and after the introduction of the report system is summarized in Table 2. The report system caused a significant decrease in the use of carbapenem as well as in the use of first-generation cephems, fosfomycin, lincomycin, and anti-MRSA drugs; however, it caused an increase in the use of fourthgeneration cephems, i.e., cefozopran and cefpirome, and second- and third-generation cephems. The introduction of the system significantly reduced the AUD for carbapenem, although the overall use of antibiotics remained unaltered. Interestingly, the AUD of both third- and fourth-generation cephems increased from 12.13 to 14.02 and from 7.77 to 10.79 , respectively. These increases may be attributed to the substitution of carbapenem antibiotics with these cephems for treating $P$. aeruginosa infections.

Fig. 4 and 5 show the changes in the susceptibility of $P$. aeruginosa to panipenem and imipenem before and after the introduction of the report system. The appearance of panipenem-resistant $P$. aeruginosa decreased significantly from $41.38 \%$ to $24.00 \%$ after the introduction of the system ( $p<0.001$; Chi-square test), while the appearance of imipenem-resistant $P$. aeruginosa decreased from $18.23 \%$ to $10.91 \%(p<0.01)$. Following the introduction of the report system, the reduced occurrence of panipenem-resistant $P$. aeruginosa was more apparent than that of imipenem-

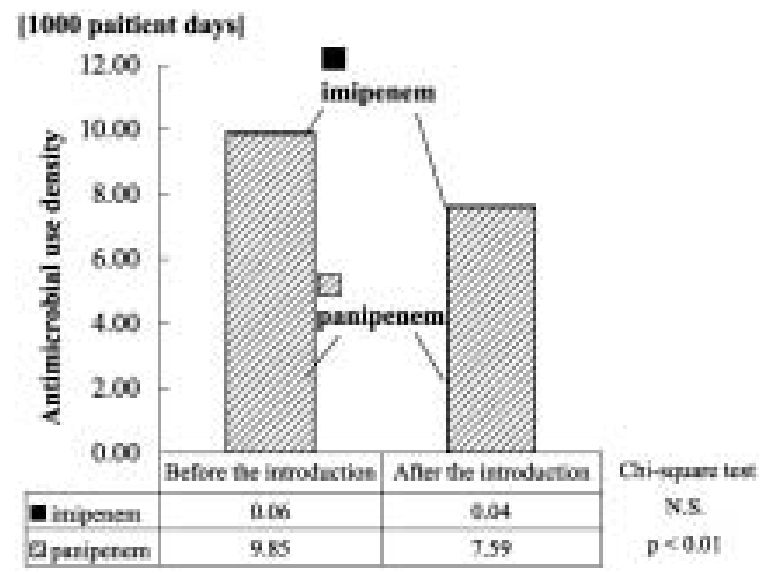

Fig 3. Comparison of the Antimicrobial Use Density for Carbapenem before and after the Introduction of the System.

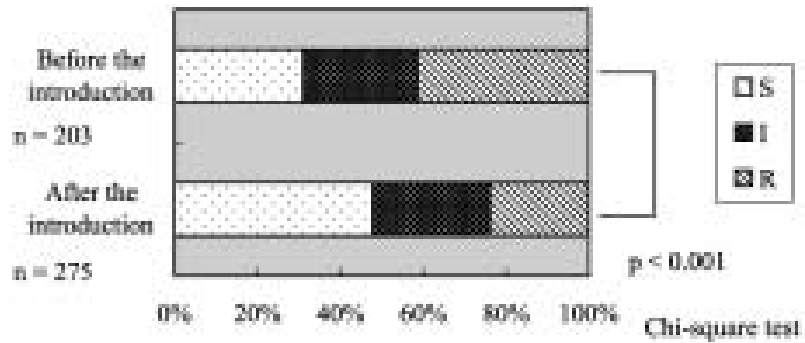

Fig .4 . Change in Susceptibility of Pseudomonas aeruginosa to Panipenem after the Introduction of the System (inpatients).

The susceptibility to antibiotics is classified as sensitive(S), resistant( $\mathrm{R})$, and intermediate( $(\mathrm{I})$ based on the guidelines proposed by the $\mathrm{Na}$ tional Committee for Clinical Laboratory Standards.

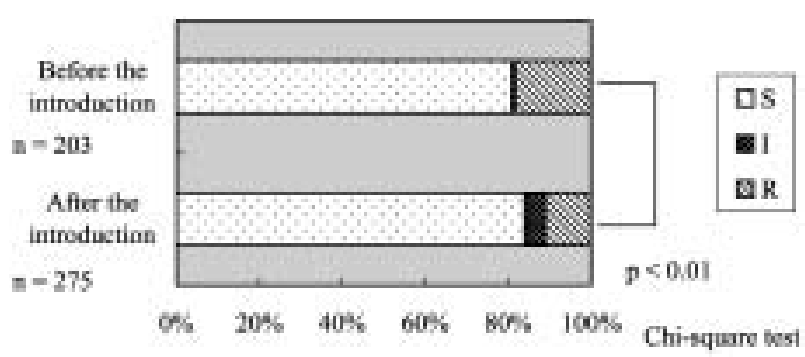

Fig .5. Change in Susceptibility of Pseudomonas aeruginosa to Imipenem after the Implementation of the System (inpatients).

The susceptibility to antibiotics is classified as sensitive(S), resistant(R), and intermediate(I) based on the guidelines proposed by the $\mathrm{Na}$ tional Committee for Clinical Laboratory Standards.

resistant $P$. aeruginosa. The reduced occurrence was proportionate to the total amounts of both carbapenems used. In our hospital, more than $95 \%$ of the total carbapenem used is panipenem; hence, there was an obvious reduction in the occurrence of panipenem-resistant $P$. aeruginosa following the introduction of the report system. As described above, following the introduction of the system, the total amount of carbapenem antibiotics used decreased, while the total amounts of penicillins and second-, third-, and fourthgeneration cephem antibiotics used increased.

Consequently, the susceptibility of $P$. aeruginosa to both piperacillin and cefoperazone decreased, as shown in Fig. 6 and 7. There was a slight increase in the susceptibility of $P$. aeruginosa to cefoperazone, which was intermediate (I), ( $p<0.05$, Fig. 6), while the susceptibility of $P$. aeruginosa to piperacillin did not change significantly. The AUD for penicillin increased from 7.35 to 7.71 and that for piperacillin, a type of penicillin, increased from 2.40 to 3.04. Be cause the amount usage of piperacillin in that of all antibiot- 


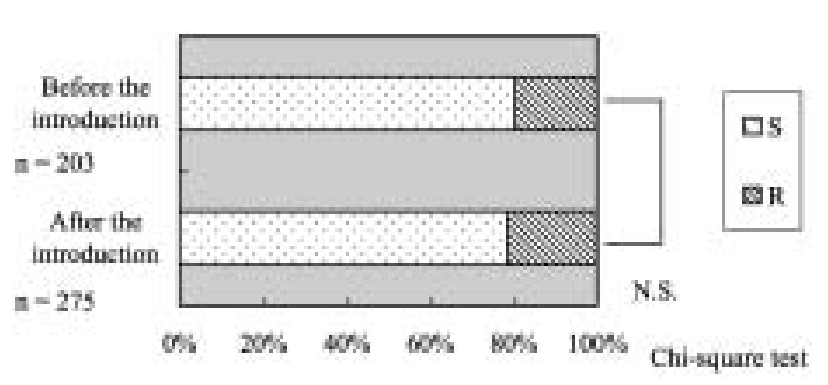

Fig .6. Change in Susceptibility of Pseudomonas aeruginosa to Piperacillin after the Implementation of the System (inpatients).

The susceptibility to antibiotics is classified as sensitive(S), resistant $(R)$, and intermediate(I) based on the guidelines proposed by the $\mathrm{Na}$ tional Committee for Clinical Laboratory Standards.

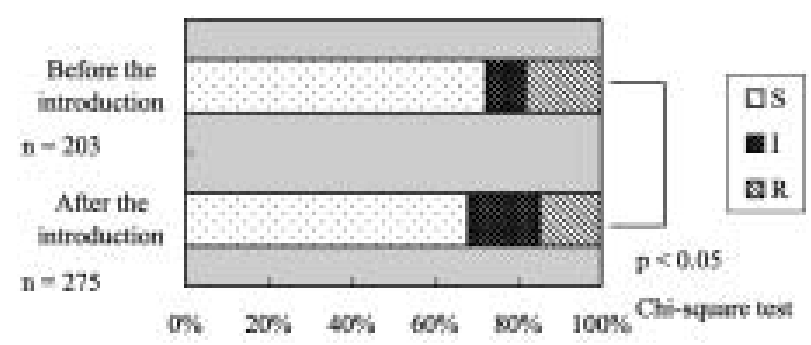

Fig .7. Change in Susceptibility of Pseudomonas aeruginosa to Cefoperazone after the Implementation of the System (inpatients).

The susceptibility to antibiotics is classified as sensitive(S), resistant(R), and intermediate(I) based on the guidelines proposed by the $\mathrm{Na}$ tional Committee for Clinical Laboratory Standards.

ics had become the result without too much a lot, there was no change in the susceptibility to this antibiotic. However, a decrease in susceptibility toward this antibiotic is predicted. The AUD for cefoperazone, a third-generation cephem, increased from 6.18 to 7.18 ; thus, there was a slight decrease in the susceptibility to cefoperazone. It should be noted that the susceptibility to cefoperazone decreases if its usage increases.

Table 3 shows the results regarding the detection of MDRP in our hospital before and after the introduction of the system. Prior to the introduction of the report system, over the past one year, MDRP was detected in $2.1 \%$ (five cases) of $233 P$. aeruginosa. However, the introduction of the system decreased the rate of MDRP to $0.66 \%$ during the same period; however, this decrease was not significant.

There are some hospitals applying the report system ${ }^{9-12)}$. The submission rate was various. $\left(40 \%{ }^{9}\right), 77.5 \%{ }^{10)}, 91.4$ $\%{ }^{11)}$, from $80 \%$ to approximately $100 \%{ }^{12)}$ ) In a past study, it was mentioned about the relation of the pharmacist to the report system as follows. If a doctor does not submit the report, a pharmacist contacts a nurse of the infection manage ment room. A pharmacist manages the report. (It is not mentioned what a pharmacist does concretely.) If a doctor does not submit the report, a pharmacist contacts a chief physician or ICD.

There is a common point in a past study ${ }^{9-12)}$. It is that submission rate is not $100 \%$. Though a report system is applied, the doctor can use the antibiotics even if the doctor does not submit a report. In this study, a pharmacist told the necessity of the report system to a doctor and the submis sion rate was $100 \%$. It is necessary for the pharmacist to maintain communication with a doctor. The pharmacist contacted all doctors who did not submit a report, and they understood it. A difference with a past study and this study is that the pharmacist contacted all doctors who did not understand the report system and led them to follow a rule.

There is the study about the introduction of the report sys tem in the broad spectrum antibiotics till now. In the past study, the total number of antibiotic vial was compared in the introduction of report system. In the neither study, AUD was used. If inpatients decrease in an investigation period, the total number of antibiotic vial decreases. If the period when the patient is hospitalized shortens, the total number decreases. It is necessary to add the number of inpatients and the hospitalization days so that we compare the use situation of the antibiotics appropriately. Therefore we used the AUD which was the value that revised the number of inpa tients and the hospitalization days. Because we calculated AUD, we were able to compare quantity of the antibiotics more appropriately.

It has been reported that the development of bacterial re sistance to antibiotics is correlated to the amount of antibiotic used, and optimization of antibiotic treatments is clearly required to prevent resistance ${ }^{13,14)}$. Antibiotic control programs have played an important role in helping prevent the emergence of bacterial resistance ${ }^{15,16)}$. In our hospital, the introduction of the report system for the use of both panipenem and imipenem has contributed enormously to the reduction in the total amount of carbapenem antibiotics used, has prevented the emergence of carbapenem-resistant

Table 3 . Detection of Multiple Drug-resistant Pseudomonas aeruginosa (MDRP) before and after the Implementation of the System.

\begin{tabular}{|c|c|c|c|c|}
\hline & P. aeruginosa & MDRP & total & Ratio of MDRP[\%] \\
\hline Before the introduction & 233 & 5 & 238 & 2.10 \\
\hline After the introduction & 299 & 2 & 301 & 0.66 \\
\hline
\end{tabular}


$P$. aeruginosa, and has reduced the occurrence of MDRP.

We plan to establish appropriate guidelines for the use of third- and fourth-generation cephem antibiotics on the basis of the present study.

\section{References}

1) D.A. Goldmann, R.A. Weinstein, R.P. Wenzel, O.C. Tablan, R.J. Duma, R.P. Gaynes, J. Schlosser, W.J. Martone, Strategies to prevent and control the emergence and spread of antimicrobial-resistant microorganisms in hospitals: a challenge to hospital leadership, JAMA, 275, 234-240 (1996).

2) K. Senda, Y. A rakawa, K. Nakashima, H. Ito, S. Ichiyama, K. Shimokata, N. Kato, M. Ohta, Multifocal outbreaks of metallo-betalactamase-producing Pseudomonas aeruginosa resistant to broad-spectrum beta lactams, including carbapenems, Antimicrob. Agents Chemother., 40, 349-353 (1996).

3) Y. Carmeli, N. Troillet, G.M. Eliopoulos, M.H. Samore, Emergence of antibiotic-resistant Pseudomonas aeruginosa : comparison of risks associated with different antipseudomonal agents, Antimicrob. Agents Chemother., 43, 1379-1382 (1999).

4) Y. Carmeli, N. Troillet, A.W. Karchmer, M.H. Samore, Health and economic outcomes of antibiotic resistance in Pseudomonas aeruginosa, Arch. Intern. Med., 159, 1127-1132 (1999).

5) B. Cao, H. Wang, H. Sun, Y. Zhu, M. Chen, Risk factors and clinical outcomes of nosocomial multi-drugresistant Pseudomonas aeruginosa infections, J. Hosp. Infect., 57, 112-118 (2004).

6) M.E. Charlson, P. Pompei, K.L. Ales, C.R. Mackenzie, A new method of classifying prognostic comorbidity in longitudinal studies development and validation, $J$. Chronic Dis., 40, 373-383 (1987).

7) D. Capella, Descriptive tools and analysis, WHO Reg.
Publ. Eur. Ser., 45, 55-78 (1993).

8) 0. Cars, S. Molstad, A. Melander, V ariation in antibiotic use in the European Union, Lancet, 357, 18511853 (2001).

9) H. Inoue, M. A sahi, T. matsunaga, Y. Katou, T. Okabe, T. Honda, Y. Y amazaki, S. Ohmori, Development of a medical surveillance communication system by providing information from the pharmacy division to the infection control team, J. Jpn. Soc. Hosp. Pharm., 43, 236-239 (2007).

10) Y. Hamaguchi, S. Oe, T. Tsuji, K. Hatanaka, A survey of proper use of injectable antibiotics, J. Jpn. Soc. Hosp. Pharm., 42, 1219-1222 (2006).

11) M. Ikemoto, T. Sasaki, Changes in the amount of antibiotic use after the introduction of the reporting system of specified antibiotics, J. Jpn. Soc. Hosp. Pharm., 43, 513-515 (2007).

12) H. Nakamura, T. Kanematsu, Y. Maeda, M. Takai, T. Ito, The status of use of antibiotics for injection at Gifu Social Insurance Hospital, J. Jpn. Soc. Hosp. Pharm., 43, 232-235 (2007).

13) R. Gaynes, The impact of antimicrobial use on the emergence of antimicrobial-resistant bacteria in hospitals, Infect. Dis. Clin. North Am., 11, 757-765 (1997).

14) J.E. Jr. McGowan, A ntimicrobial resistance in hospital organisms and its relation to antibiotic use, Rev. Infect. Dis., 5, 1033-1048 (1983).

15) J.E. Jr. McGowan, Do intensive hospital antibiotic control programs prevent the spread of antibiotic resis tance?, Infect. Control Hosp. Epidemiol ., 15, 478-483 (1994).

16) R.W. Coleman, L.C. Rodondi, S. Kaubisch, N.B. Granzella, P.D. O'Hanley, Cost-effectiveness of prospective and continuous parenteral antibiotic control : experience at the Palo A Ito Veterans Affairs Medical Center from 1987 to 1989, Am. J. Med., 90, 439-444 (1991). 